\title{
Risk Assessment Method of Automotive Product Injury in China
}

\author{
Yongqin Feng ${ }^{1, ~ a, ~ Y a n ~ Y i n ~}{ }^{2, b}$, Zhenhua $\mathrm{Cao}^{3, \mathrm{c}}$ and Xiaorui Zhang ${ }^{4, \mathrm{~d}}$ \\ 1,2,4 Defective Product Administrative Center of AQSIQ, Beijing, 100191, China \\ ${ }^{3}$ Beijing General Research Institute of Nonferrous Metals, Beijing, 100088, China \\ afengyq@dpac.gov.cn, byiny@dpac.gov.cn, 'caozhenhua_science@163.com, \\ zhangxr@dpac.gov.cn
}

Keywords: automotive product injury; risk assessment; early warning grade

Abstract. Automotive product injury risk evaluation is an effective means to reducing the loss caused by automotive product injuries by controlling the risks of such injuries. Based on the product injury monitoring data from the national product injury information monitoring system, and focusing on potential injury severity and injury occurrence possibility, the paper proposed FRS analysis paradigm of risk assessment of automotive product injury and further discussed the division of early warning grade of the injury, as well as carried out risk assessment and early warning analysis on China's automotive products.

\section{Introduction}

Automotive industry is an important part of the current world economy. According to the statistics of China Association of Automobile Manufacturers (CAAM), since 2009, China's automobile production and sales have consecutively maintained the top position across the world for 5 years. However, while automobiles become a part of the consumers' daily life, safety problems caused by defective automobile products occur from time to time. In the meantime, the automotive product injury cases have also increased gradually, endangering the customer's personal and property safety. In order to prevent and resolve the product injury problems, it is urgently needed that the research on the automotive product injury risk assessment should be carried out in our country.

At present, the product injury risk assessment is gradually drawing attention from the academic circles. [1] have set up the injury cost model (ICM) for the nonfatal injury assessment of consumer products, based on the injury data from the United State's NEISS and 17 large database. [2] proposed the principles, procedures and contents of consumer products assessment in China, and the suggestions on how to drive the consumer products risk assessment forward. [3] summarized the management modes of consumer products safety risk assessment and the related research developed in the developed countries of the world. [4] presented the injury scenes and 3D matrix integration method of consumer products risk assessment. Overall, it can be concluded that the research on the automotive product injury risk assessment is still in the weak state, and that the performed researches are mainly focused on the consumer products injury risk assessment.

The paper is organized as follows. After the automotive product risk assessment method is proposed and studied in regard to the special research on the risk assessment method, the risk assessment and early warning are made for automotive product injury in China, based on the product injury monitoring data from the national product injury information monitoring system.

\section{Assessment Method of Automotive Product Injury Risk}

The automotive product injury risk assessment is a quantization assessment process, through which the possibility of automotive injuries' resulting effects or losses is evaluated, with the potential gravity of injury and the possibility of injuries considered as the key in the automotive product injury assessment; and in which, the special research into the risk assessment method is made to build the FRS Analysis Mode for automotive product injury risk assessment (where, $\mathrm{F}$ : the frequency of injuries occurring in a specific time period; R: the time interval between the latest injury and the analysis day; S: the potential 
gravity of injury, viz. the grade of injury), with the credit card analysis ideas selected and used for reference, and the focus put on the potential gravity of injury and the possibility of injuries.

With the reference to the National Product Injuries Monitoring Report Card, there are three values available for the grade of injury (VC_SHYZCD): 1, 2 or 3, of which, 1 responds to the minor injury, 2 the medium injury and 3 the serious injury. Thus, the grade of injury (VC_SHYZCD) is decomposed into three variables as blow:

$$
\begin{gathered}
S_{1}=\left\{\begin{array}{lc}
1 & \text { Serious injury } \\
0 & \text { Others }
\end{array}\right. \\
S_{2}=\left\{\begin{array}{lc}
1 & \text { Medium injury } \\
0 & \text { Others }
\end{array}\right. \\
S_{3}=\left\{\begin{array}{lc}
1 & \text { Minor injury } \\
0 & \text { Others }
\end{array}\right.
\end{gathered}
$$

According to the FRS Analysis Mode, all automotive products for which the serious injury even occurs once need early warning. Moreover, if it is simply setting enough weight for $S_{01}$, other products which also need early warning will be covered. So, based on the main factor method, the SAS application is used to make the main factor analysis on scored data, with the characteristic values decomposed, and the weights distributed to different indexes in regard to the characteristic values of vectors in different $F, R, S_{02}, S_{03}$ vector groups. The weights can be determined in the following formula:

$$
\omega_{j}=\frac{\lambda_{j}}{\sum_{j=1}^{4} \lambda_{j}}, j=1,2,3,4
$$

where, $\lambda_{j}$ is the characteristic root of the $\mathrm{j}^{\text {th }}$ factor, $\omega_{j}$ is the number of weights of the $\mathrm{j}^{\text {th }}$ factor .

The early warning index can be calculated from the following formula based on the weighted mean method:

$$
\text { Index }_{i}=\sum_{j=1}^{5}\left(\text { weight }_{j} \times V A R_{i j}\right)
$$

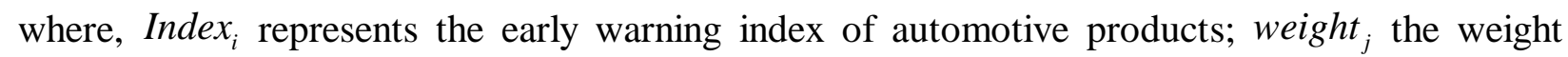
corresponding with the $\mathrm{j}^{\text {th }}$ indicator (variable); $V A R_{i j}$ the score of the $\mathrm{j}^{\text {th }}$ indicator.

In the analysis of automotive product injury risk assessment, the early warning index can be obtained from the following formula:

$$
\text { Index }=\omega_{1} \cdot F+\omega_{2} \cdot R+W \cdot S_{01}+\omega_{3} \cdot S_{02}+\omega_{4} \cdot S_{03}
$$

The grade of automotive product injury early warning is based on the early warning score, presenting early warnings of different grades on the basis of the product injury early warning scores. With reference to the weather early warning signal, the product injury early warning is divided into such 4 grades as Blue, Yellow, Orange and Red, of which the gravity becomes more serious in turn, that is, Blue represents the lowest grade of early warning; and Red the highest grade of early earning. A Red early warning is released when the score of product injury early warning is not lower than 90; an Orange one is released when the score of product injury early warning is lower than 90 but not lower than 80; a Yellow one is released when the score of product injury early warning is lower than 80 but not lower than 70; and a Blue one is released when the score of product injury early warning is between 0 and 70. 


\section{Risk Assessment and Early Warning Results}

During the period from January 1 of 2014 to December 31 of 2014, there were 15846 person-times of effective automotive product injury monitored totally by the national product injury information monitoring system. The calculated results from the automotive product injury risk assessment show an average interval of 5.71 days from the analysis day to the day when latest automotive product injury occurred in 2014. Also, the calculation gives the following results: an average injuries of 185.86, an average serious injuries of 8.71, an average medium injuries of 47 , as well as an average minor injuries of 130.29 and an average warning score of 75.35 .

Tab. 1 The Descriptive Statistics of Total Injury Warning Score in 2014

\begin{tabular}{ccccccc}
\hline Variable & Label & N & $\begin{array}{c}\text { Mean } \\
\text { Values }\end{array}$ & $\begin{array}{c}\text { Standard } \\
\text { Deviation }\end{array}$ & $\begin{array}{c}\text { Min. } \\
\text { Values }\end{array}$ & Max. Value \\
\hline R & Injury interval & 7 & 5.71 & 10.00 & 1.00 & 28.00 \\
F & Injury frequency & 7 & 185.86 & 371.52 & 5.00 & 1025.00 \\
S01 & Serious injury & 7 & 8.71 & 14.00 & 0.00 & 39.00 \\
S02 & Medium injury & 7 & 47.00 & 89.82 & 0.00 & 249.00 \\
S03 & Minor injury & 7 & 130.29 & 268.21 & 4.00 & 737.00 \\
score & Warning score & 7 & 75.35 & 27.48 & 39.61 & 100.00 \\
\hline
\end{tabular}

In 2014, based on the warning score, the top three automotive product subcategories were cars, with the average warning score of 100.00; trucks, with the average warning score of 99.99; small passenger cars, with the average warning score of 99.98. With regard to the product injury frequency, the top three automotive product subcategories were cars, with the average product injury frequency of 1025.00; trucks, with the average product injury frequency of 98.00; small passenger cars, with the average product injury frequency of 65 . In addition, as to the number of serious injuries, the top three automotive product subcategories were cars, trucks and small passenger cars in turn, with the number of average serious injuries reaching 39,10.00 and 9.00 separately.

Tab. 2 The Descriptive Statistics of Warning Score of Automotive under Subcategories

\begin{tabular}{|c|c|c|c|c|c|c|}
\hline No. & $\begin{array}{l}\text { Automotive Product } \\
\text { Subcategories }\end{array}$ & $\mathrm{F}$ & S01 & S02 & S03 & Score \\
\hline 1 & Cars & 1025.00 & 39.00 & 249.00 & 737.00 & 100.00 \\
\hline 2 & $\begin{array}{l}\text { Large and medium } \\
\text { passenger cars }\end{array}$ & 62.00 & 2.00 & 22.00 & 38.00 & 84.06 \\
\hline 3 & Trucks & 98.00 & 10.00 & 33.00 & 55.00 & 99.99 \\
\hline 4 & Small passenger cars & 65.00 & 9.00 & 14.00 & 43.00 & 99.98 \\
\hline 5 & $\begin{array}{l}\text { Vehicular parts and } \\
\text { accessories }\end{array}$ & 40.00 & 0.00 & 10.00 & 30.00 & 40.60 \\
\hline 6 & Automobile trailers & 5.00 & 0.00 & 1.00 & 4.00 & 39.61 \\
\hline 7 & Others & 6.00 & 1.00 & 0.00 & 5.00 & 63.18 \\
\hline
\end{tabular}

In the automotive product subcategories, the top three products cars, trucks and vans of the highest scores in turn get the grade of automotive product early warning Red; buses get the grade Orange; and police cars/vans, vehicle doors, and semi-trailers the grade Blue. 
Tab.3 The Warring Scores of Product Injuries with the Highest Scores in Automobile

Subcategories

\begin{tabular}{|c|c|c|c|c|c|c|c|c|}
\hline Rank & $\begin{array}{c}\text { Product } \\
\text { Subcategory }\end{array}$ & Product & $\mathbf{F}$ & $\mathbf{R}$ & S01 & S02 & S03 & Score \\
\hline 1 & Cars & Car & 1025 & 1 & 39 & 249 & 737 & 100.00 \\
\hline 2 & Trucks & Cargo vehicle & 98 & 1 & 10 & 33 & 55 & 99.99 \\
\hline 3 & Small passenger cars & Van & 65 & 2 & 9 & 14 & 43 & 99.98 \\
\hline 4 & $\begin{array}{l}\text { Large and medium } \\
\text { passenger cars }\end{array}$ & Bus & 62 & 1 & 2 & 22 & 38 & 84.06 \\
\hline 5 & Others & Police van/car & 6 & 6 & 1 & 0 & 5 & 63.18 \\
\hline 6 & $\begin{array}{l}\text { Vehicular parts and } \\
\text { accessories }\end{array}$ & Door & 40 & 1 & 0 & 10 & 30 & 40.60 \\
\hline 7 & Automobile trailer & Semi-trailer & 5 & 28 & 0 & 1 & 4 & 39.61 \\
\hline
\end{tabular}

\section{Conclusion}

The formulation of scientific automotive product risk assessment methods is an effective way to prevent the automotive products from injuries and release in time the early warning for automotive product injuries. In this paper, FRS Analysis Mode is presented, and furthermore, the product injury early earning is initially divided into such 4 grades as Blue, Yellow, Orange and Red, based on the automotive product injury data from the national product injury information monitoring system. Also, the safety state of automotive products in China is assessed. All the stated is of great significance to the development of automotive products safety monitoring in China. With the continuous improvement on the data quality and the data items, the automotive product injury risk assessment methods will be modified and improved unceasingly.

\section{Acknowledgements}

This work was financially supported by the Science and Technology Program of General Administration of Quality Supervision, Inspection and Quarantine titled Three-dimensional Finite Element Model for Chest and Abdomen of Crash Test Dummies by Taking 50th Percentile Adult Male as Example (No. 2014QK219).

\section{References}

[1] B.A. Lawrence, T.R. Miller, A.F. Jensen, et al.:Injury Control and Safety Promotion, 7(2000)

[2] Xia Liu, Hongqi Luo: China Quality, 9(2009) (In Chinese)

[3] Luyao Lin, Wanjin Tang, Zhongxue Li et al.: Journal of Safety Science and Technology, 10(2014) (In Chinese)

[4] Jing Chen, Yan Yan, Huiyuan Sun et al. : Standard \& Quality of Light Industry, 5(2013) (In Chinese) 